\title{
Impactos da Pandemia na Sustentabilidade da Mobilidade Urbana no Período Pandêmico
}

Impacts of the Pandemic on the Sustainability of Urban Mobility in the Pandemic Period Impactos de la Pandemia en la Sostenibilidad de La Movilidad Urbana en el Período Pandémico

Ketryan B. Martins

Graduanda, UNESP, Brasil ketryan.martins@unesp.br

Luzenira Alves Brasileiro Professora Doutora, UNESP, Brasil luzenira.brasileiro@unesp.br 


\title{
Revista Científica ANAP Brasil
}

\author{
ISSN 1984-3240 - Volume 14, número 35, 2021
}

\section{RESUMO}

O objetivo do trabalho é analisar a influência da pandemia na mobilidade da cidade das pessoas entrevistadas e nos impactos referentes a sustentabilidade dessas cidades no período pandêmico. Ele foi desenvolvido com base em uma coleta de dados por entrevista a usuários dos sistemas de transportes de diferentes cidades do Brasil, a qual foi realizada de forma remota utilizando formulário aplicado pela plataforma Google Forms, sendo que, após a tabulação dos dados, foi analisada a quantidade de pessoas que utilizavam o transporte público e que passaram a utilizar outros modos de locomoção, além do tempo de viagem para cada passageiro; e com isso, foi feito o estudo do caso. Por meio dos resultados obtidos na pesquisa, encontrou-se que o uso do modo automóvel, a pé e motocicleta aumentaram, obtendo o valor a mais de $9 \%, 5 \%$ e $2 \%$, respectivamente; porém o uso da bicicleta permaneceu sendo de $5 \%$ dentre as pessoas que responderam o formulário. Além disso, foi possível obter que $41 \%$ das pessoas que se transportavam por menos de 30 minutos de automóvel seriam possivelmente pessoas que poderiam aderir o uso da bicicleta, caso existissem incentivos governamentais, o que deixaria a cidade mais sustentável. Os resultados mostram que a convergência dos dados das diferentes cidades indica um consenso da população brasileira de que há a necessidade de se manter a segurança nos sistemas de mobilidade urbana, pois é um serviço básico necessário à toda a população.

\section{ABSTRACT}

The aim of this work is to analyze the influence of the pandemic on the mobility of the people interviewed in the city and on the impacts related to the sustaintability of these cities during the pandemic period. It was developed based on data collection by interviewing users of transport systems in different cities in Brazil, which was carried out remotely using a form applied by the Google Forms platform. After tabulating the data, the number of people who used public transport and started to use other modes of locomotion were analyzed, in addition to the travel time for each passenger; and thus, the case study was carried out. Through the results obtained in the research, it was found that the use of automobile, on foot and motorcycle increased, obtaining the value of $9 \%, 5 \%$ and $2 \%$, respectively, more than previously; however, the use of bicycles remained at 5\% among the people who answered the form. In addition, it was possible to obtain that $41 \%$ of people who traveled for less than 30 minutes by car would possibly be people who could use the bicycle, if there were government incentives, which would make the city more sustainable. The results show that the convergence of data from different cities indicates a consensus among the Brazilian population that there is a need to maintain security in urban mobility systems, as it is a basic service needed by the entire population.

\section{RESUMEN}

El objetivo del trabajo es analizar la influencia de la pandemia en la movilidad de las personas entrevistadas en la ciudad y en los impactos relacionados con la sostenibilidad de estas ciudades durante el período pandémico. Se desarrolló a partir de una recopilación de datos mediante entrevistas a usuarios de sistemas de transporte en diferentes ciudades de Brasil, que se realizó de forma remota mediante un formulario aplicado por la plataforma Google Forms. Luego de tabular los datos, se analizó el número de personas que utilizaban transporte y que empezaron a utilizar otros modos de locomoción, además del tiempo de viaje de cada pasajero; y con eso se llevó a cabo el estudio de caso. A través de los resultados obtenidos en la investigación, se encontró que aumentó el uso del automóvil, en modo a pie y en motocicleta, obteniendo el valor de más del $9 \%, 5 \%$ y $2 \%$, respectivamente; sin embargo, el uso de bicicletas se mantuvo en un $5 \%$ entre las personas que contestaron el formulario. Además, se pudo obtener que el $41 \%$ de las personas que viajan menos de 30 minutos en automóvil posiblemente sean personas que podrían usar la bicicleta, si existieran incentivos gubernamentales, que harían la ciudad más sustentable. Los resultados muestran que la convergencia de datos de diferentes ciudades indica un consenso entre la población brasileña de que existe la necesidad de mantener la seguridad en los sistemas de movilidad urbana, ya que es un servicio básico que necesita toda la población.

PALAVRAS-CHAVE: Mobilidade Urbana. COVID-19. Poluição. 


\section{Revista Científica ANAP Brasil}

ISSN 1984-3240 - Volume 14, número 35, 2021

\section{INTRODUÇÃO}

\subsection{Mobilidade Urbana e COVID-19}

A mobilidade urbana é o resultado da interação dos fluxos de deslocamento de pessoas e bens no espaço urbano (MINISTÉRIO DAS CIDADES, 2005), a qual vem tendo fortes modificações desde meados do século passado, o que é consequência principalmente de um processo de urbanização, crescimento desorganizado das cidades e uso maior do transporte motorizado individual pela população (IPEA, 2010a apud Ipea, 2016a).

Atualmente, essa mobilidade vem apresentando mudanças cada vez maiores, devido ao advento da doença COVID-19. A doença "coronavirus disease 2019", com sigla COVID-19, está causando grandes impactos na população mundial (CRUZ, 2020).

Em janeiro de 2020, a Organização Mundial da Saúde (OMS) declarou a Emergência de Saúde Pública de Interesse Internacional devido ao vírus (CRUZ, 2020) e pandemia em março. No mês seguinte, o COVID-19 Map - John Hopkins Hospital (2020) contabilizou 1.181.825 casos confirmados no mundo, 63.902 mortes e 244.246 casos curados (apud SANT'ANA, 2020). Já em 9 de julho de 2021, às $12 \mathrm{~h} 22$, foram contabilizados no mundo, também segundo o COVID-19 Map - Johns Hopkins Coronavirus Resource Center (2021), 185.715.975 casos e 4.013.136 mortes; e no Brasil, de acordo com o Portal do COVID-19 (2021), até o dia 8 de julho de 2021 às 18h10, ocorreram 18.962.762 casos confirmados, 17.422 .854 recuperados e 530.179 mortes.

A transmissão do vírus ainda é algo que está sendo estudado, mas sabe-se que pessoas que entram em contato com cerca de 1 metro de alguém com os sintomas e/ou entra em contato com secreções contaminadas, correm o risco de contrair o vírus (MORAIS, 2020).

Além disso, essas pessoas também podem adquirir a doença por pessoas assintomáticas (MORAIS, 2020). Uma das medidas adotadas para evitar o contágio da doença é o isolamento social, que limita a circulação das pessoas e consequentemente altera os planos de mobilidade urbana.

Nesse contexto, o transporte público, por exemplo, representa um ambiente de alto risco de contágio do vírus por causa do grande número de pessoas dentro dele, pouco espaço de ventilação de ar e por ter vários lugares no transporte que podem abrigar o vírus que são bastante tocados, como a catraca, braços de assento, entre outros (UITP, 2020 apud LIMA; SCHECHTMAN; BRIZON; FIGUEIREDO, 2020).

Um estudo chinês relata que pessoas viajando de ônibus podem ser infectadas por outros passageiros sentados a mais de 4,5 metros de distância e esse mesmo estudo constatou que o vírus continua dentro de ônibus por mais de 30 minutos (SOUTH CHINA MORNING POST, 2020 apud LIMA; SCHECHTMAN; BRIZON; FIGUEIREDO, 2020).

Sendo assim, as pessoas que precisam sair de casa por alguma razão, acabam tendo que buscar outros meios de transporte possíveis. Logo, as que tem maiores condições financeiras dentre as que utilizam o transporte público, podem acabar buscando como alternativa automóveis particulares, já que poucas pessoas utilizam veículos não motorizados, muito devido ao fato de que as cidades não incentivam a isso (MINISTÉRIO DAS CIDADES, 2005). 


\subsection{Sustentabilidade nos Transportes Urbanos}

Há mais de sessenta anos, as pessoas se deslocavam na maioria das cidades do Brasil por transportes públicos coletivos (Ipea, 2016b). Com o avanço da indústria automotiva no país, os deslocamentos motorizados individuais acabaram crescendo e esses transportes coletivos acabaram diminuindo, o que em termos de sustentabilidade, foi algo bastante negativo, já que o sistema foi alterado para que privilegiasse os deslocamentos privados (Ipea, 2016b).

Desta forma, ainda segundo Ipea (2016b), o aumento das taxas de motorização privadas no país fez com que aumentasse os poluentes globais e locais. Por meio da Figura 1 é possível observar a evolução das emissões de $\mathrm{CO}_{2}$ e a projeção que foi feita para o ano de 2020, considerando uma situação sem pandemia.

Figura 1 - Emissões de $\mathrm{CO}_{2}$ pelos veículos automotores no Brasil (em $10^{6}$ de $\mathrm{CO}_{2}$ )

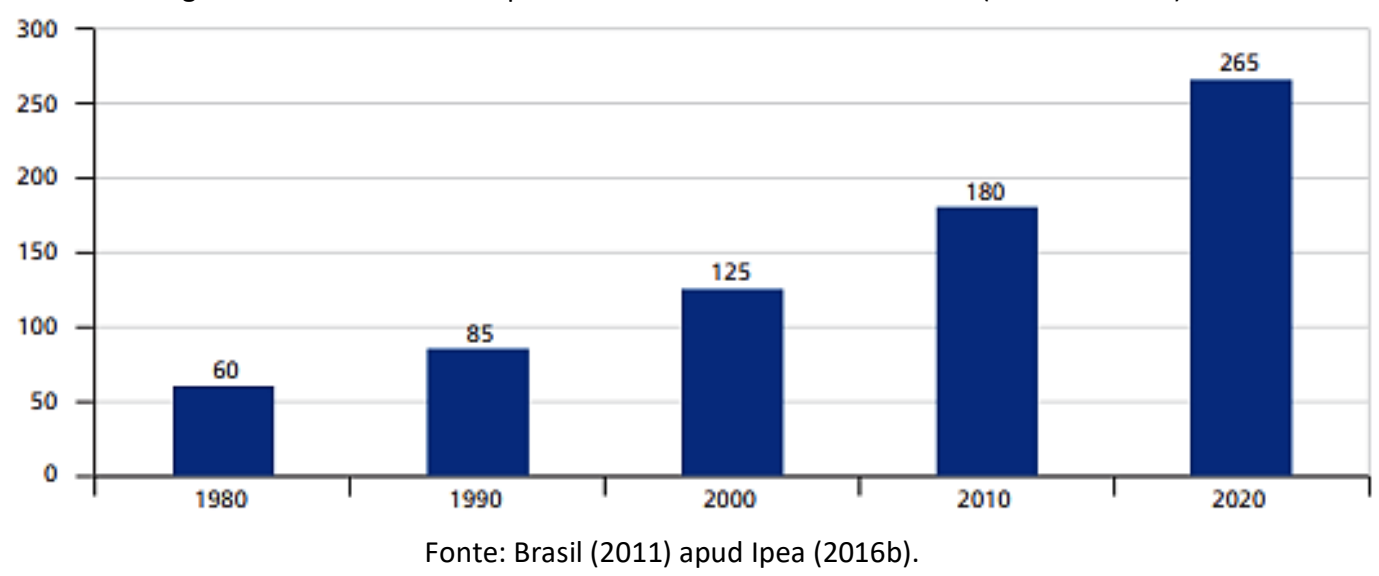

Pela Figura 2, pode-se verificar as Emissões de $\mathrm{CO}_{2}$ das modalidades de transporte urbano por passageiro e quilômetro.

Figura 2 - Emissões de $\mathrm{CO}_{2}$ das modalidades de transporte urbano por passageiro e quilômetro

\begin{tabular}{lcccc}
\hline \multirow{2}{*}{$\begin{array}{l}\text { Modalidade de } \\
\text { transporte }\end{array}$} & $\begin{array}{c}\text { Emissōes } \\
\text { quilométricas }\end{array}$ & $\begin{array}{c}\text { Ocupação } \\
\text { média veicular }\end{array}$ & Emissōes/passageiro km & Indice de emissão \\
\cline { 2 - 5 } & $\mathrm{kg} \mathrm{CO} / \mathrm{km}$ & Passageiros & $\mathrm{kg} \mathrm{CO} /$ passageiro km & (metró=1) \\
\hline Metrõ & 3,16 & 900 & 0,0035 & 1,0 \\
Onibus & 1,28 & 80 & 0,0160 & 4,6 \\
Automóvel & 0,19 & 1,50 & 0,1268 & 36,1 \\
Motocicleta & 0,07 & 1,00 & 0,0711 & 20,3 \\
Veizulos pesados & 1,28 & 1,50 & 0,8533 & 243,0 \\
\hline
\end{tabular}

Fonte: Ipea (2011).

Com isso, percebe-se que é necessário que sempre tenha uma preocupação de aumentar a participação do transporte público coletivo, já que, por exemplo, o ônibus tem índice de emissão de $\mathrm{CO}_{2}$ de 4,6 e o automóvel de 36,1.

O incentivo governamental do uso da bicicleta poderia ser uma alternativa para que a cidade ficasse mais sustentável, o que resultará na redução da emissão de poluentes atmosféricos, da poluição sonora e de vibrações e a redução da dependência de recursos não renováveis (FABIANO, 2016). Além disso, outra alternativa seria o incentivo do transporte público em detrimento do individual, oferecendo melhores condições de uso deles, como menor aglomeração, maior frota e diminuição do valor da condução do transporte (Ipea, 2021a). 


\section{Revista Científica ANAP Brasil}

ISSN 1984-3240 - Volume 14, número 35, 2021

\section{OBJETIVOS}

O objetivo do trabalho é analisar a influência da pandemia na mobilidade da cidade das pessoas entrevistadas e nos impactos referentes a sustentabilidade dessas cidades no período pandêmico.

\section{METODOLOGIA}

O trabalho foi desenvolvido com base em uma coleta de dados por entrevista a usuários dos sistemas de transportes em diferentes cidades de todo o Brasil, sendo elas: Andradina (SP), Aparecida do Taboado (MS), Brasília (DF), Campinas (SP), Campos dos Goytacazes (RJ), Carangola (MG), Caxias (MA), Ceilândia (DF), Curitiba (PR), Diadema (SP), Elesbão Veloso (PI), Embu Guaçu $(\mathrm{SP})$, Formosa (GO), Fortaleza (CE), Foz do Iguaçu (PR), Franca (SP), Ibitinga (SP), Ilha Solteira (SP), Itapecerica da Serra (SP), Itu (SP), Jaboatão dos Guararapes (PE), Jundiaí (SP), Lages (SC), Manaus (AM), Montenegro (RS), Novo Oriente do Piauí (PI), Osasco (SP), Palhoça (SC), Passos (MG), Paulo de Faria (SP), Picos (PI), Pinheiro Machado (RS), Praia Grande (SP), Recife (PE), Salvador (BA), Santa Maria (RS), Santana de Parnaíba (SP), São Bernardo do Campo (SP), São José do Rio Preto (SP), São Miguel (RN), São Paulo (SP), Suzano (SP), Taboão da Serra (SP), Tatuí (SP), Taubaté (SP), Teresina (PI), Ubaitaba (BA), Uberlândia (MG), Valença do Piauí (PI) e Votuporanga (SP).

A coleta de dados foi realizada de forma remota. O material utilizado na coleta de dados foi um formulário aplicado pela plataforma Google Forms, que foi dividido em duas partes.

A primeira etapa consistiu em questões a respeito das formas pelas quais eram realizados os transportes do entrevistado antes da pandemia e seus motivos. A segunda parte consistiu em perguntas sobre mesmo assunto, porém depois de iniciada a quarentena.

Esse formulário foi enviado por redes sociais e as informações foram coletadas no final de maio de 2020 até o final de junho de 2020. Os dados foram filtrados, tabulados com o auxílio do software Microsoft Office Excel e, posteriormente, plotados em gráficos.

Após a tabulação dos dados, foi analisada a quantidade de pessoas que utilizavam o transporte público e que passaram a utilizar outros modos de locomoção, além do tempo de viagem para cada passageiro, para assim, ser feito o estudo do caso. 


\section{RESULTADOS E DISCUSSÕES}

Dentre as 232 respostas obtidas, foi possível perceber que $47,8 \%$ das pessoas entrevistadas estão precisando sair de casa por algum motivo durante a quarentena.

$\mathrm{Na}$ Figura 3 pode-se observar o transporte que essas pessoas utilizavam antes da pandemia.

Figura 3 - Modo de transporte que os entrevistados utilizam normalmente

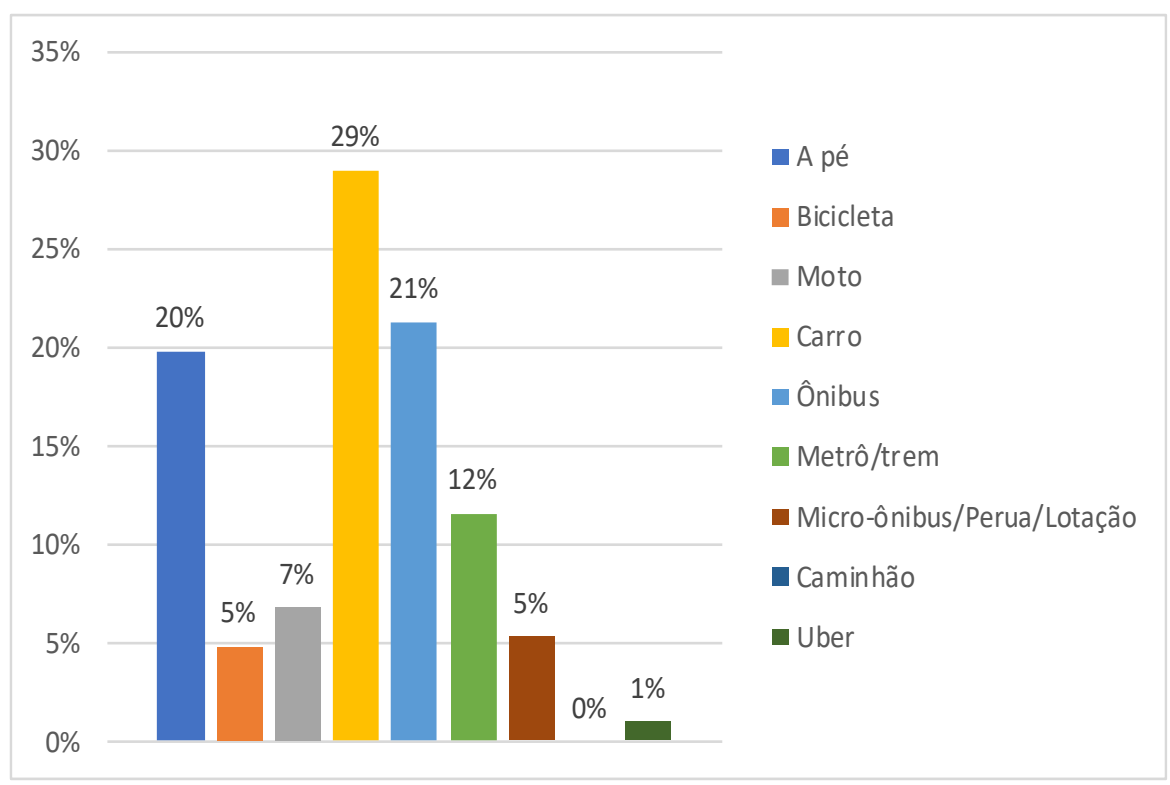

Fonte: Elaborado pelo próprio autor (2020).

Já na Figura 4, é possível verificar o transporte que essas mesmas pessoas estão utilizando durante a pandemia.

Figura 4 - Modo de transporte que os entrevistados utilizam durante a pandemia

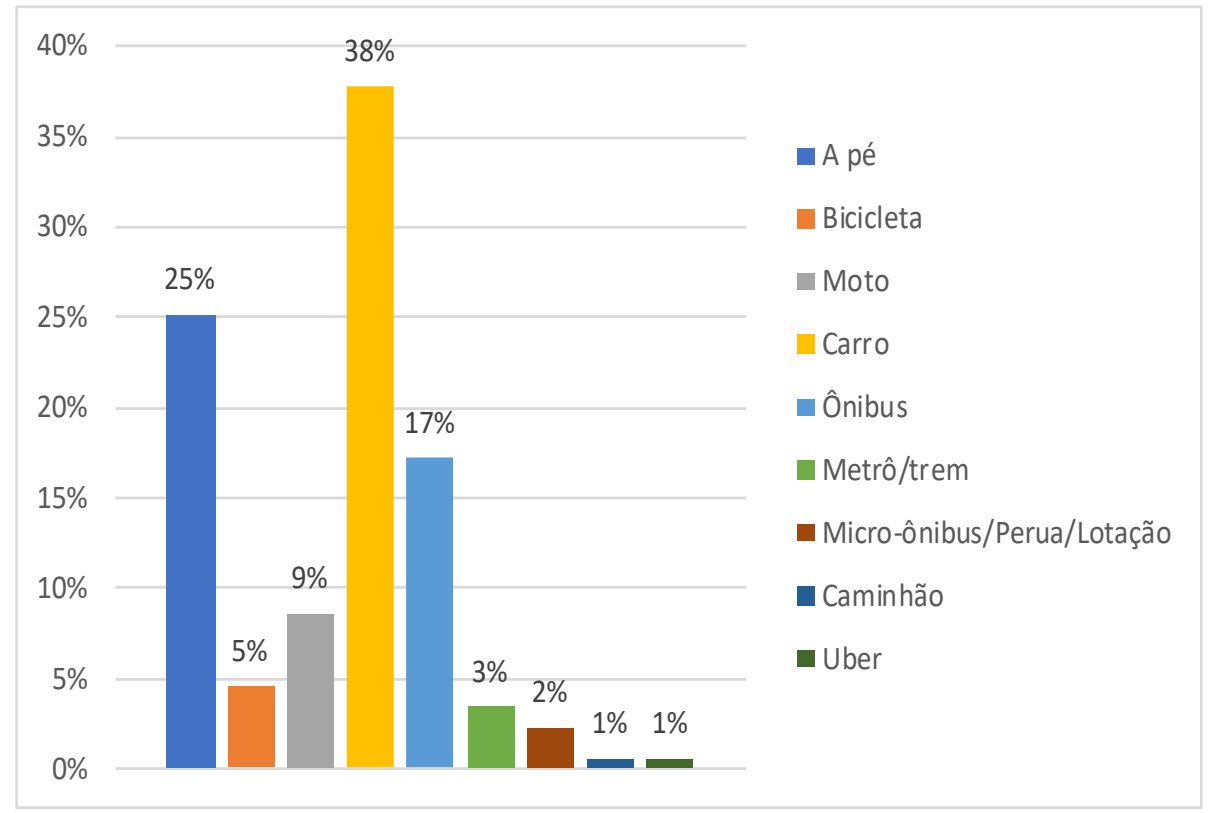

Fonte: Elaborado pelo próprio autor (2020). 


\section{Revista Científica ANAP Brasil}

ISSN 1984-3240 - Volume 14, número 35, 2021

Com isso, percebe-se analisando a Figura 3 e a Figura 4, que o uso do modo automóvel e motocicleta aumentaram, tendo aumento de $9 \%$ e $2 \%$, respectivamente.

Mesmo com o aumento da utilização de automóvel e motocicleta, houve uma redução no uso de combustível nessa época de pandemia, pois, ao comparar as vendas de combustíveis utilizados no transporte no mês de março de 2020 com as vendas no mesmo mês de 2019, foi reduzido o volume de todos combustíveis vendidos, com exceção do óleo diesel (AZEVEDO et al., 2020).

Pela pesquisa aqui presente, é possível ver que o uso do modo a pé aumentou, tendo um acréscimo de $5 \%$ no valor, porém o uso de bicicleta permaneceu o mesmo que foi calculado anteriormente a pandemia, igual a $5 \%$.

Se caso a utilização do automóvel e motocicletas não tivesse crescido, seria possível dizer que, como o modal a pé aumentou, possivelmente este seria um dos motivos da diminuição do uso do combustível, já que para essas pessoas, poderia ser um modo mais viável no momento. Porém, não foi isso que ocorreu, então não se pode confirmar que o consumo do combustível diminuiu por essa razão.

Como muitas pessoas não estavam saindo de casa no final de maio de 2020 até o final de junho de 2020, provavelmente a diminuição do uso do combustível ocorreu devido ao fato de que, mesmo com o aumento de pessoas usando transportes privados, essa quantidade de pessoas não atingiu a como era antes.

Analisando-se melhor sobre a bicicleta, a Figura 5 permite verificar o tempo aproximado da viagem que os entrevistados estão enfrentando em seus deslocamentos na pandemia.

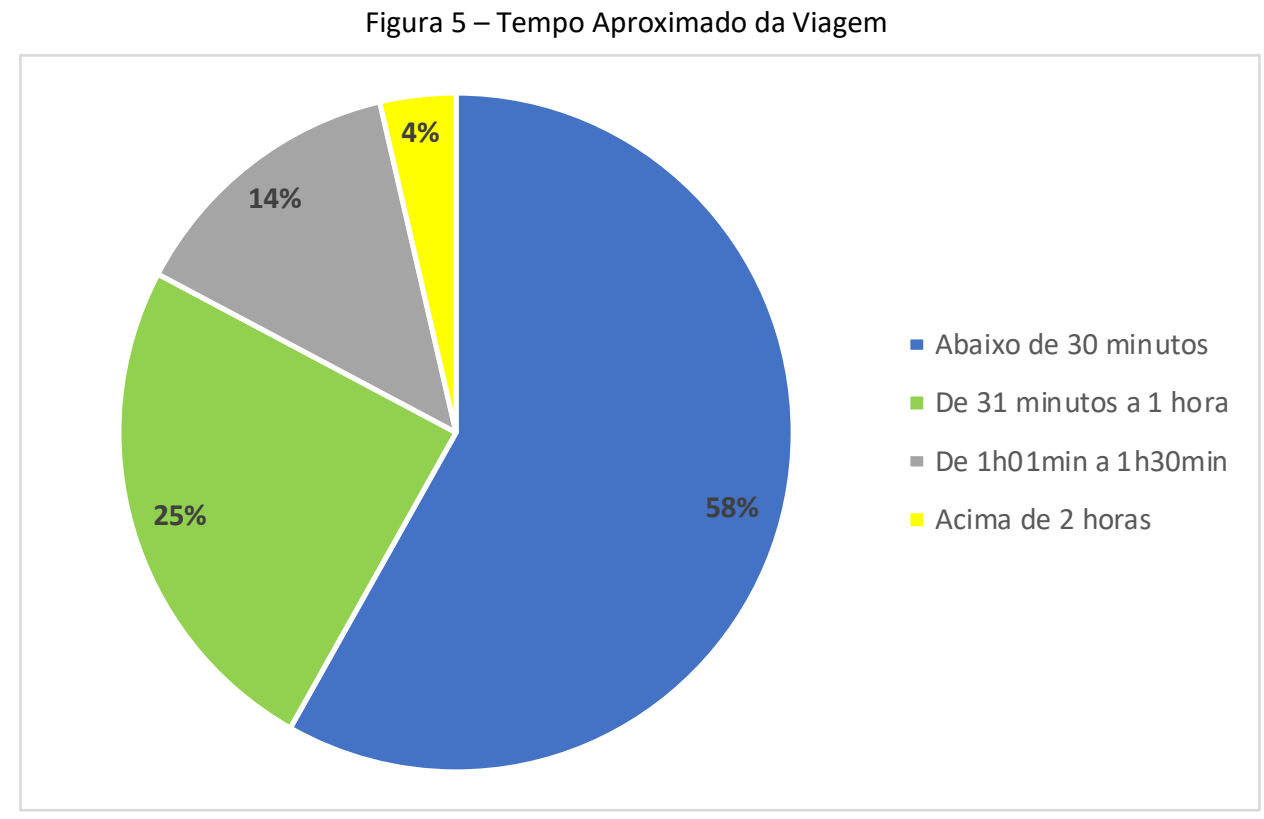

Fonte: Elaborado pelo próprio autor (2020).

Como pode-se perceber, $58 \%$ das pessoas se transportam com tempo de viagem abaixo de 30 minutos. Com isto, pela Figura 6, obtém-se a porcentagem de pessoas entrevistadas que usam algum determinado modo de transporte com viagem abaixo de 30 minutos. 
Figura 6 - Modo de transporte que os entrevistados utilizam em viagem abaixo de 30 minutos

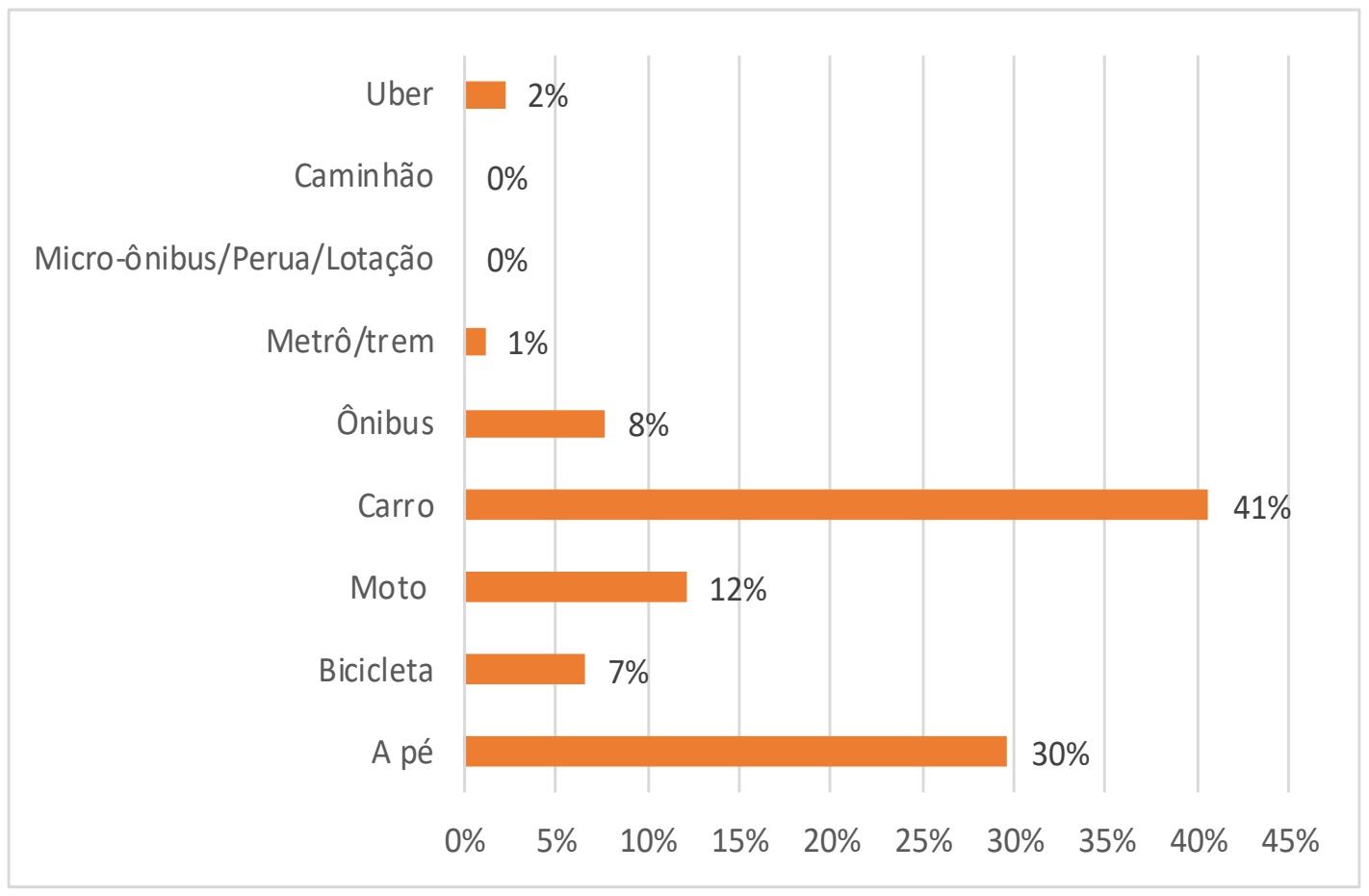

Fonte: Elaborado pelo próprio autor (2020).

Sendo assim, $41 \%$ das pessoas que se transportam por menos de 30 minutos de automóvel seriam possivelmente pessoas que poderiam aderir o uso da bicicleta, caso tivesse incentivo e vias que possibilitassem a utilização.

O LabCidade - Laboratório Espaço Público e Direito à Cidade (2019), publicou em seu site sobre uma das dificuldades que os ciclistas de São Paulo encontram, que é a de estacionar as bicicletas, pois, segundo a pesquisa que fizeram, a maioria das respostas reclamaram da quantidade de vagas ser insuficiente.

Na Figura 7, os problemas encontrados pelos ciclistas da pesquisa ao utilizar os bicicletários estão expostos. Vale ressaltar que no caso desta pergunta, os participantes do questionário poderiam selecionar múltiplas respostas, o que fez com que as porcentagens ultrapassassem valor total de $100 \%$. Portanto, os números do gráfico dizem respeito à quantidade de participantes, dentre o total deles, selecionou determinada alternativa (LABCIDADE FAU USP, 2019). 
Revista Científica ANAP Brasil

ISSN 1984-3240 - Volume 14, número 35, 2021

Figura 7 - Problemas encontrados ao utilizar os bicicletários

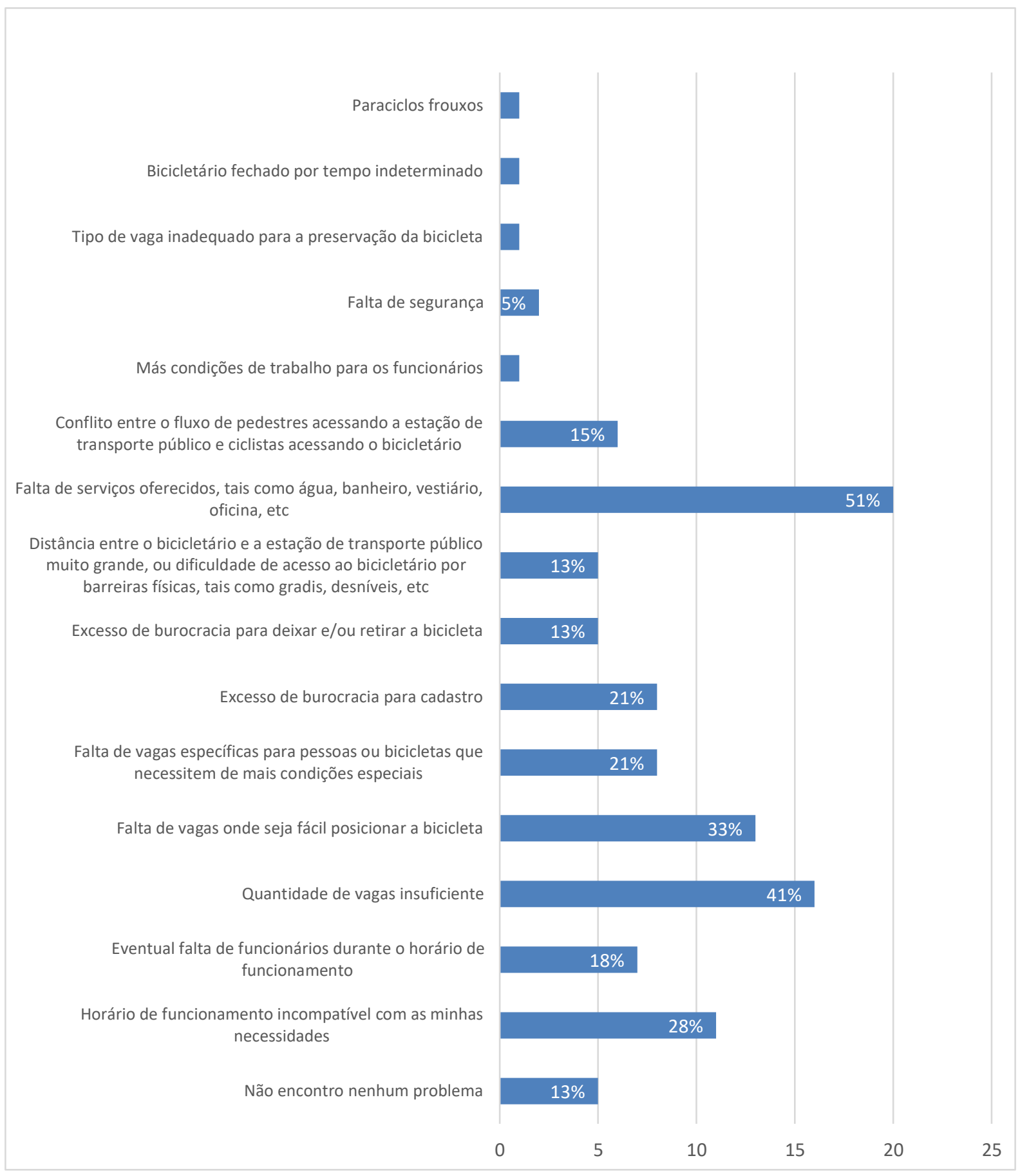

Fonte: LabCidade (Laboratório Espaço Público e Direito à Cidade) - Faculdade de Arquitetura e Urbanismo da Universidade de São Paulo (2019). 


\section{Revista Científica ANAP Brasil}

ISSN 1984-3240 - Volume 14, número 35, 2021

\section{CONCLUSÃO}

Por meio dos valores encontrados na pesquisa, pode-se obter, como já foi citado, que o uso do modo automóvel e motocicleta aumentaram, obtendo acréscimo de $9 \%$ e $2 \%$, respectivamente; porém o uso da bicicleta permaneceu o mesmo, sendo de $5 \%$ dentre as pessoas que responderam o formulário, o que pode ter acontecido devido aos diversos problemas que ocorrem com bicicletários e nas ciclovias.

Encontrou-se também que $41 \%$ das pessoas entrevistadas se transportavam por menos de 30 minutos de automóvel. Como é um tempo mais curto, essas pessoas poderiam, talvez, aderir o uso da bicicleta, caso existissem incentivos governamentais, o que deixaria a cidade mais sustentável.

A questão do aumento dos veículos privados motorizados é algo que deve ser levada em conta ao pensar na situação da mobilidade urbana no período de pandemia, pois a decisão dessas pessoas que começaram a utilizar esses modos são tomadas devido a diferentes fatores. Logo, quanto mais veículos particulares houver nas ruas, o impacto de poluição será cada vez maior, além de que, também gerará mais congestionamento.

Por fim, a pesquisa teve o intuito de obter um cenário amplo sobre a realidade atual de diferentes cidades brasileiras. Então, a amostra é composta por dados de diferentes cidades do Brasil.

A convergência do resultado referente aos dados das diferentes cidades indica um consenso da população brasileira de que há a necessidade de se manter a segurança nos sistemas de mobilidade urbana, tendo em vista que se trata de um serviço básico e, portanto, necessário à toda a população.

\section{REFERÊNCIAS}

AZEVEDO, Tasso; BARCELLOS, Felipe; TSAI, David; CREMER, Marcelo; GAUDERETO, Felipe; COLUNA, Iris; ALBUQUERQUE, Igor; ALENCAR, Ane; ZIMBRES, Bárbara; BRANDÃO JR, Amintas; COSTA JR, Ciniro; PIATTO, Marina; QUINTANA, Gabriel; POTENZA, Renata. Impacto da Pandemia de Covid-19 nas Emissões de Gases de Efeito Estufa no Brasil. SEEG Brasil, maio de 2020. Disponível em: https://www.oc.eco.br/wp-content/uploads/2020/05/SEEGOC_Nota_Tecnica_Covid19_Final.pdf. Acesso em: 15 jul. 2021.

CARVALHO, Carlos Henrique Ribeiro de. Desafios da Mobilidade Urbana no Brasil. Ipea - Instituto de Pesquisa Econômica Aplicada, Brasília, 2021a. Disponível em: http://repositorio.ipea.gov.br/bitstream/11058/6664/1/td_2198.pdf. Acesso em: 15 jul. 2021.

CARVALHO, Carlos Henrique Ribeiro de. Emissões Relativas de Poluentes do Transporte Urbano. Ipea - Instituto de Pesquisa Econômica Aplicada, jun. $2011 . \quad$ Disponível em: <http://repositorio.ipea.gov.br/bitstream/11058/5574/1/BRU_n05_emiss\%C3\%B5es.pdf>. Acesso em: 15 Jul. 2021.

CARVALHO, Carlos Henrique Ribeiro de. Mobilidade Urbana Sustentável: Conceitos, Tendências e Reflexões. Ipea Instituto de Pesquisa Econômica Aplicada, Brasília, 2016b. Disponível em: http://repositorio.ipea.gov.br/bitstream/11058/6637/1/td_2194.pdf. Acesso em: 19 jul. 2021.

Coronavírus Brasil. Painel Coronavírus. Disponível em: https://covid.saude.gov.br/. Acesso em: 9 Jul. 2021.

COVID-19 Map - Johns Hopkins Coronavirus Resource Center. Johns Hopkins Coronavirus Resource Center. Disponível em: https://coronavirus.jhu.edu/map.html. Acesso em: 9 Jul. 2021. 


\section{Revista Científica ANAP Brasil}

ISSN 1984-3240 - Volume 14, número 35, 2021

CRUZ, Roberto Moraes et al. COVID-19: emergência e impactos na saúde e no trabalho. Rev. Psicol., Organ. Trab., Brasília, v. 20, n. 2, p. I-III, jun. $2020 . \quad$ Disponível em http://pepsic.bvsalud.org/scielo.php?script=sci_arttext\&pid=S1984-66572020000200001\&lng=pt\&nrm=iso. Acesso em 15 jul. 2021. http://dx.doi.org/10.17652/rpot/2020.2.editorial.

FABIANO, Maria Lucia Alves. A Mobilidade Urbana e o Papel da Bicicleta como Indutor de Inclusão Social e de Transformação da Cidade. Estratégias de Projeto e Intervenção nas Metrópoles Contemporâneas: Experiências e Perspectivas - Universidade Presbiteriana Mackenzie, nov. 2016. Disponível em: https://www.mackenzie.br/fileadmin/OLD/62/ARQUIVOS/PUBLIC/SITES/PORTAL/IV_COLOQUIO_BRASILPORTUGAL/25.pdf. Acesso em: 15 jul. 2021.

Lima, G. C. L. S., Schechtman, R., Brizon, L. C., Figueiredo, Z. M. (2020, Abril de 2020). Transporte público e COVID-19. $\mathrm{O}$ que pode ser feito?. Rio de Janeiro. Centro de Estudos em Regulação e Infraestrutura da Fundação Getúlio Vargas (FGV CERI). Disponível em: https://ceri.fgv.br/sites/default/files/publicacoes/202005/covid_e_mobilidade_urbana_0.pdf. Acesso em: 15 jul. 2021.

Mobilidade e política urbana: subsídios para uma gestão integrada /Coordenação de Lia Bergman e Nidia Inês Albesa de Rabi. - Rio de Janeiro: IBAM; Ministério das Cidades, 2005. Disponível em: https://static.fecam.net.br/uploads/695/arquivos/1156134_Mobilidade_Urbana__BAM.pdf. Acesso em: 15 jul. 2021.

MORAIS, Protásio de. Conheça os principais grupos de risco para a Covid-19 e saiba como prevenir a doença. Governo do Mato Grosso, 17 jul. 2021. Disponível em: http://www.mt.gov.br/-/13958350-conheca-os-principais-grupos-derisco-para-a-covid-19-e-saiba-como-prevenir-a-doenca. Acesso em: 15 jul. 2021.

SANT'ANA, Geisa; IMOTO, Aline Mizusaki; AMORIM, Fábio Ferreira; TAMINATO, Monica; PECCIN, Maria Stella; SANTANA, Levy Aniceto; GÖTTEMS, Leila Bernarda Donato; CAMARGO, Erika Barbosa. Infecção e óbitos de profissionais da saúde por COVID-19: revisão sistemática. Acta Paul Enferm, v. 33, eAPE20200107, ago. 2020. Disponível em: https://acta-ape.org/wp-content/uploads/articles_xml/1982-0194-ape-33-eAPE20200107/19820194-ape-33-eAPE20200107.pdf. Acesso em: 31 jul. 2021.

ZERON, Vicente Sisla; HARKOT, Marina. Ciclovias não bastam: as dificuldades enfrentadas por ciclistas de São Paulo para estacionar as bicicletas. LabCidade (Laboratório Espaço Público e Direito à Cidade) - Faculdade de Arquitetura e Urbanismo da Universidade de São Paulo, 15 out. 2019. Disponível em:

http://www.labcidade.fau.usp.br/ciclovias-nao-bastam-as-dificuldades-enfrentadas-por-ciclistas-de-sao-paulo-paraestacionar-as-bicicletas. Acesso em: 15 jul. 2021. 